\title{
Orientación Académica y Profesional: percepción del mercado de trabajo desde las Consejerías de Educación españolas ${ }^{1}$
}

\author{
David Doncel Abad² \\ ORCID: http://orcid.org/0000-0002-9399-2904 \\ Sonsoles San Román Gago ${ }^{3}$ \\ ORCID: http://orcid.org/0000-0003-3114-4720 \\ Carlos Vecina Merchante ${ }^{4}$ \\ ORCID:http://orcid.org/0000-0001-8439-8455
}

\section{Resumen}

La combinación de un mercado de trabajo poco solidario con los jóvenes y la implantación de un modelo de orientación académica y profesional cada vez más propenso a resaltar la dimensión vocacional dibuja un contexto que arroja importantes interrogantes sobre cómo se conectan uno y otro. En consecuencia, se estima importante conocer cómo se percibe la relación entre ambos factores en el proceso de orientación vocacional de los estudiantes en el sistema educativo español. Para alcanzar este fin general se han fijado los siguientes subobjetivos: analizar la imagen que se otorga al mercado laboral según contexto socioeducativo; comprender cómo se percibe la función de orientación académica y profesional según contexto socioeducativo; y elaborar una tipología de modelos de orientación Académica y Profesional según contexto socioeducativo. La técnica aplicada ha consistido en la entrevista orientada de manera inducida. El estilo analítico adoptado se centra en los casos. Se hace hincapié en la exposición e interpretación de los relatos caso a caso, siguiendo una doble lógica: una de análisis comparativo dentro de cada perfil o within case analysis y otra de análisis cruzado de casos pertenecientes a distintos perfiles o cross-case analysis. Se aplica un modelo interpretativo fundamentado en el Método Comparativo Constante, pues permite descubrir conceptos y relaciones y organizarlos en esquemas teóricos explicativos. Los resultados alcanzados permiten conjeturar que existen tres percepciones del mercado de trabajo extraídas de la relación de éste con el sistema educativo según contexto socioeducativo, y que están influyendo en el proceso de Orientación Académica y Profesional.

\section{Palabras clave}

Orientación profesional - Educación secundaria - Vocación.

1- Este artículo se ha elaborado con material del proyecto de I+D+l titulado Contexto socioeconómico y Orientación Educativa y profesional del profesorado financiando por el Ministerio de Economía y Competitividad. Referencia: CS02013-47168-R.

2- Universidad de Salamanca, Salamanca, España. Contacto: davidoncel@usal.es.

3- Universidad Autónoma de Madrid, Madrid, España. Contacto: sonsosan@ucm.es.

4- Universidad de las Islas Baleares, España. Contacto: carlos.vecina@uib.es.

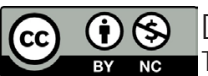




\section{Academic and vocational guidance: perception of labor market from the Spanish Autonomous Offices of Education}

\section{Abstract}

The combination of labor market unfavorable to young people and the implementation of an academic and vocational guidance model, focused on the vocational dimension, portraits a context that raises important questions about how they connect with each other. Consequently, we consider it important to know how the relationship between both factors is perceived during the vocational guidance process in the Spanish educational system. To achieve this general goal, we have set the following sub-objectives: to analyze the image assigned to the labor market according to the socio-educational context; to understand how the function of academic and professional guidance is perceived according to the socio-educational context, and to build a typology of academic and professional guidance models according to the socio-educational context. The chosen technique is the interview oriented in a induced way. The analytical style focuses on the cases. Emphasis is on the exposition and interpretation of the stories on a per case basis, following a two-fold logic: the logic within each case and the cross-case analysis. In addition, an analytical strategy is applied, related to the Constant Comparative Method, which allows to discover concepts and relationships and organize them in explanatory theoretical schemes. The results leads to speculate that there are three perceptions of the labor market based on the perceived relationship between the labor market and the education system according to a given socio-educational context, and that these interpretations influence the process of academic and vocational guidance.

\section{Keywords}

Vocational guidance - Secondary Education - Vocation.

\section{Planteamiento}

La globalización ha producido un nuevo escenario de relaciones sociales, económicas y territoriales que han afectado directamente el mercado de trabajo. La intensificación de la mundialización económica está generando pautas de crecimiento del subempleo y del incremento del desempleo juvenil que afectan tanto a las sociedades del Sur como a las del Norte del planeta (UNESCO, 2015). Por ejemplo, una de las consecuencias sociales más graves de la Gran Recesión fue el incremento de las tasas de paro, que si en Europa aumentaron de un modo importante, en el caso español azotaron con fuerza a la población juvenil.

5-This article is elaborated with information of the I+D+I project entitled: Socioeconomic Context and Educational and Vocational Guidance for Teachers, financed by the Ministry of Economy and Competitiveness. Reference: CS02013-47168-R. 
La relación entre el mercado de trabajo y la población joven en España se ha caracterizado por una singular precariedad. Según los datos del Observatorio de las Ocupaciones (2016), para el año 2006, la tasa de paro juvenil alcanzó un 13,80\%, y, en 2012, ascendió a su valor máximo de los últimos 10 años, con un 42,73\%. Aunque descendiera a un 35,15\% en 2015, desterraba al desempleo a uno de cada tres jóvenes. Es más, durante la última década las tasas de paro juvenil nunca descendieron de las dos cifras, poniendo de manifiesto la fragilidad del vínculo entre educación formal y empleo.

Efectivamente, la deficiente inserción laboral de los jóvenes españoles ha respondido más a patrones estructurales que a coyunturales, aunque agravados por las dinámicas globales. Un elemento explicativo clave, aquí, estriba en cómo se han conectado las habilidades requeridas para el desempeño de la ocupación con la formación obtenida en las aulas, pues se observan claros desajustes entre una y otra. A modo de ilustración, según los datos recogidos en la Encuesta de Población Activa, en el año 2015, un 68\% de los ocupados menores de 25 años desempeñó un trabajo para el que se requerían menos habilidades de las poseídas. Y lejos de ser un problema en remisión, desde 2013 a 2016 , la sobre cualificación creció más de un 12\%. En el trasfondo de este fenómeno late el problema de la distribución de la población según su formación en el mercado de trabajo y, en particular, tratándose de los jóvenes, de cómo se produce la transición desde el aula al empleo. Esta cuestión apunta directamente hacia las propias funciones sociales que debe cumplir el sistema educativo español en general y a la de orientación educativa y profesional en particular (MARTÍNEZ; PÉREZ; MARTÍNEZ, 2014).

La concreción formal de la orientación educativa y profesional comenzó con la Ley General de Educación (LGE) de 1970. Pero será con la Ley de Ordenación General del Sistema Educativo (LOGSE) de 1990, cuando se sustanciará su integración y normalización en la educación no universitaria (BENAVENT, 2002). En su Artículo 60, se recoge una concepción de la orientación educativa y profesional vinculada al principio de Atención a la Diversidad e inspirada en el cuerpo teórico del Movimiento de la Carrera. Mediante este enfoque se pretendía, entre otros objetivos, estrechar la relación entre sistema educativo y mercado de trabajo a través de la orientación. Desde entonces se marcará un punto de referencia para la práctica docente de orientación (VELAZ DE MEDRANO et al., 2001).

A partir de este momento, y a lo largo de las sucesivas leyes orgánicas, la orientación educativa y profesional evolucionará de una configuración circunscrita mayoritariamente al ámbito educativo (GRAÑERAS; PARRAS, 2008) a potenciar su vertiente vocacional. A instancia de la Unión Europea, tras la aprobación de la Ley Orgánica de Educación de 2006 y la Ley Orgánica para la Mejora de la Calidad Educativa de 2013, se reforzarán los principios de la Educación para la Carrera (GONZÁLEZ, 2003) y la Orientación Académica y Profesional (ÁLVAREZ, 2012; ECHEVERRÍA; MARTÍNEZ, 2015) como elemento de ajuste entre formación y ocupación (CHISVERT, 2014).

A día de hoy, el debate académico en torno a cómo se conectan escuela y mercado de trabajo se encuentra vigente y abierto. Desde el ámbito internacional, se ha abordado desde diferentes dimensiones. Por ejemplo, en los de trabajos de Wang y otros autores (2014) y Tian y otros autores (2014) se analiza la función de orientación vinculada al abandono educativo. En Beich y Walden (2014) se pone el punto de atención en las 
transiciones y en la Formación Profesional. En Lore y otros autores (2014) se hace hincapié en los programas de orientación profesional. Y ahondado en las trayectorias profesionales en Secundaria destacan los trabajos de Molgat, Deschenaux y Leblanc (2011), en Canadá, y de Kim, Brown y Fong (2016) y Knight (2015), ambos en China.

En España también se encuentran contribuciones vigentes como las de Cejudo (2017), que aborda las competencias docentes en la labor de Orientación. También la de Velaz de Medrano, Manzano y Blanco (2011) donde analiza comparativamente, a nivel autonómico, los distintos modelos de orientación implementados en las distintas Comunidades Autónomas. Teniendo como objeto de estudio la atención a la diversidad y la orientación destaca el trabajo de De la Fuente (2010). Y propiamente sobre la orientación profesional en Secundaria se encuentran los estudios de Olivares, De León y Huertas y Gutiérrez (2010) y Santana, García y Cruz (2010).

Sin embargo, aunque existe una abundante investigación al respecto, se ha detectado una laguna con relación a las aportaciones desde una perspectiva sociológica sobre el estudio de la relación entre el mercado de trabajo y el sistema educativo mediado por la Orientación Académica y Profesional. A pesar de que la tendencia normativa actual apuesta por la fuente sociológica al incluir los principios del Movimiento de Educación para la Carrera en el currículo (GRAÑERAS; PARRAS, 2008; EURYDCE, 2012).

El Movimiento de Educación para la Carrera concibe la Orientación Académica y Profesional como un elemento funcionalista de encaje entre escuela y mercado de trabajo (GONZÁLEZ, 2003; SUPER; HALL, 1978), adjudicando al docente la función de distribución del alumnado entre cualificaciones y de asignación entre posiciones sociales (ÁLAVAREZ, 2012; HOYT, 1977). No obstante, en este punto, es importante matizar la objetividad de la función docente de orientación supuesta bajo este enfoque. El profesorado no actúa en un vacío cultural o social; implícito en la propia orientación se vehiculan elementos culturales o sociales, entendidos como elementos que condicionan la actividad docente. De este modo, a pesar de que según los principios del Movimiento de Educación para la Carrera la actuación del profesorado se debe guiar por criterios universalistas, dicha labor no queda limitada a la objetividad de las calificaciones, sino que se encuentra mediada por diferentes factores sociales y culturales presentes en su contexto. Por ejemplo, desde una Consejería de Educación se puede planificar un modo de orientación más equitativo o meritocrático según la singularidad de su entorno y este condicionará en última instancia la acción docente de orientación. En consecuencia, para aprehender cómo se conecta sistema educativo y mercado de trabajo mediado por la Orientación Académica y Profesional hay que poner el punto de atención tanto en el profesorado como en los factores que influyen en su acción.

Partiendo de esta premisa, analizar la percepción que desde las Consejerías se tiene sobre la relación entre el mercado de trabajo y el sistema educativo vinculado a la función docente Orientación Académica y Profesional se presenta como el eje vertebrador de esta investigación. Para alcanzar este fin general se fijan, a su vez, los siguientes sub-objetivos: estudiar la imagen que se otorga al mercado laboral según contexto socioeducativo; comprender cómo se percibe la función de orientación académica y profesional según contexto socioeducativo, y elaborar una tipología de modelos de Orientación Académica y Profesional según contexto socioeducativo. 


\section{Metodología}

\section{Muestra de casos de estudio}

Para la realización de esta investigación se han seleccionado intencionalmente diez Comunidades Autónomas (CCAA) ${ }^{6}$ : Andalucía (AND), Baleares (BAL), Canarias (CAN), Castilla y León (CyL), La Rioja (LR), Madrid (MAD), Murcia (MUR), Navarra (NAV), País Vasco (PV) y Valenciana (VAL). El elemento central ha sido considerar la confluencia de tres variables como son: el sector productivo identificador del territorio, el abandono temprano de la enseñanza y el paro juvenil. La condición para formar parte del estudio es el hecho de que dichas CCAA cubrieran los diferentes perfiles resultados de esa confluencia de factores.

Un elemento central de la investigación consiste en conocer cómo se plantea la orientación académica y profesional según contexto socioeconómico y educativo desde las Consejerías de Educación, considerando esta institución como la que marca las directrices de orientación y la formación docente en los centros de enseñanza. Veamos la presencia de esas tres variables: el peso del sector productivo en la distribución de la población ocupada,, las tasas de abandono escolar temprano y las tasas de paro juvenil.

El primer indicador se operacionaliza según el peso de la ocupación en cada uno de los sectores de actividad económica: Primario, Industrial, Construcción y Servicios. En España predomina el sector servicios, si bien con los pesos relativos dependiendo de la zona geográfica estos difieren. Ante esta diversidad, se ha optado por la siguiente clasificación según el peso de aquel sector que singulariza más al caso de estudios con relación a los otros. Por ejemplo, AND y MUR destacan por contar con un sector agrícola con un peso superior a un $8 \%$ (ver cuadro 1$)$.

Cuadro 1- Distribución de los casos de estudio según peso del sector de actividad económica

\begin{tabular}{|c|c|}
\hline $\begin{array}{l}\text { Perfil } \\
\text { Actividad Económica }\end{array}$ & CCAA \\
\hline Primario (>8\%) & AND y MUR. \\
\hline Industrial (>15\%) & CyL, NAV, LR, PV y VAL. \\
\hline Servicios (>80\%) & MAD, BAL y CAN. \\
\hline
\end{tabular}

Fuente: Elaboración propia a partir de los datos de la Encuesta de Población Activa (EPA). Instituto Nacional de Estadística (2013).

Junto a la actividad económica se añaden las tasas de paro juvenil y de abandono educativo temprano, con el fin de ajustar el perfil de los casos de estudio. Para ambas magnitudes se ha calculado la media para la población entre 18 y 24 años de edad (periodo: 2006-2015).

6- Una comunidad autónoma es una entidad territorial administrativa española, organizada en distintas conserjerías, que, dentro del ordenamiento jurídico constitucional estatal, cuenta con cierta autonomía legislativa con representantes propios, y de determinadas competencias ejecutivas y administrativas. 
Cuadro 2- Media de la tasa de desempleo juvenil y de la tasa de abandono educativo temprano por Comunidades Autónomas

\begin{tabular}{|c|c|c|}
\hline & MediaD & MediaAET \\
\hline AND & 47,60 & 32,8 \\
\hline BAL & 38,20 & 34,9 \\
\hline CAN & 46,60 & 29,9 \\
\hline CyL & 36,50 & 22,5 \\
\hline $\mathrm{LR}$ & 34,4 & 22,8 \\
\hline MAD & 36,00 & 22,2 \\
\hline MUR & 40,90 & 32,4 \\
\hline NAV & 31,40 & 14,7 \\
\hline PV & 34,30 & 12,5 \\
\hline VAL & 41,4 & 27,1 \\
\hline
\end{tabular}

Fuente: Elaboración propia a partir de los datos del Instituto Nacional Estadística (Periodo 2006-2015).

En el Gráfico 1 se muestra la distribución final de los casos según las tres variables seleccionadas, donde se observan tres tendencias grupales. Por un lado, un conjunto o perfil 1 que muestra las mayores tasas de abandono educativo temprano y de paro juvenil, conformado por todos los casos del sector primario AND y MUR y algunos del sector industrial VAL y del sector servicios BAL y CAN. Por otro, el siguiente perfil 2 muestra las tasas intermedias en ambas magnitudes, donde se agrupan los casos del sector industrial CyL y LR más uno del sector servicios MAD. Finalmente, dos casos del sector industrial de NAV y PV registran las tasas más bajas en ambos indicadores, conformando el perfil 3.

Gráfico 1- Distribución de los casos según tasa media de paro, tasa media de abandono educativo temprano y perfil de actividad económica

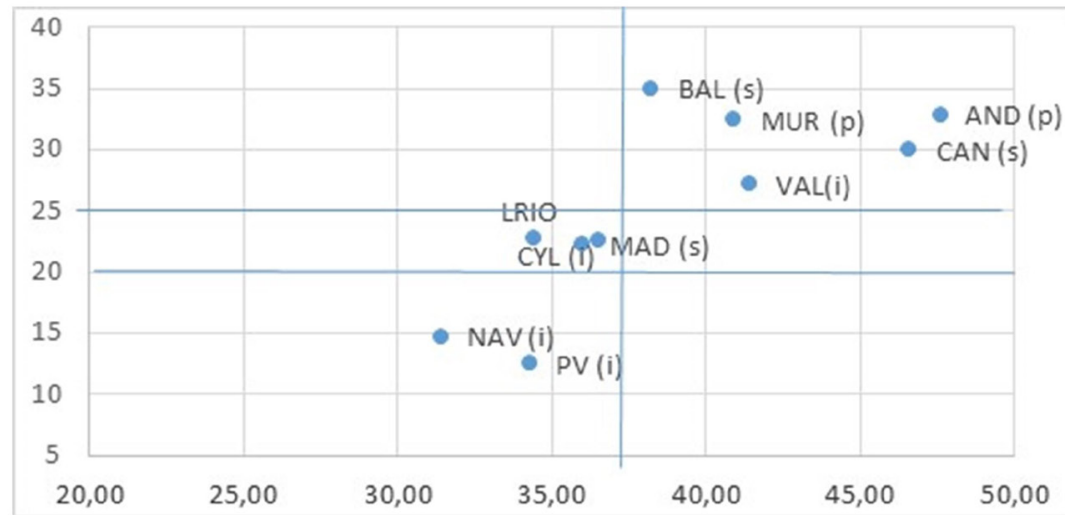

Nota: (i) Industrial, (s) Servicios y (p) Primaria.

Fuente: Elaboración propia a partir de los datos ofrecidos por el Instituto Nacional Estadística. (INE). Años 2006-2015. 
Mapa 1- Distribución geográfica de los casos de estudio según perfil socioeducativo de estudio. España.

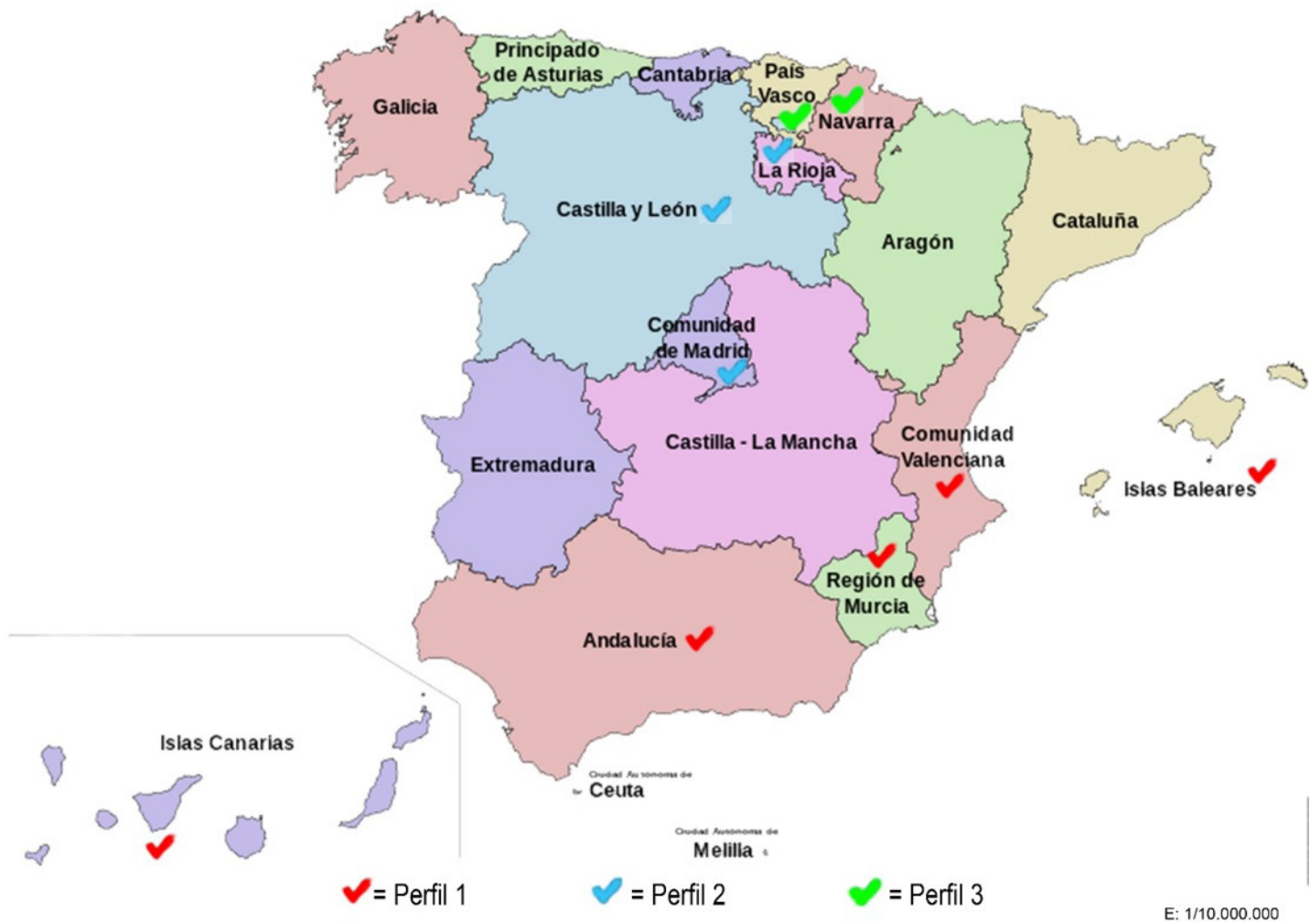

Fuente: Elaboración propia a partir de los datos ofrecidos en Galería de Mapas, Fotos e Imágenes Satélite GIFEX ${ }^{7}$.

\section{Discursos}

En el mapa 1 se muestra la distribución geografía de las CCAA seleccionadas, indicando el perfil socioeducativo al que pertenecen cada una de ellas.

En coherencia con los objetivos marcados, se seleccionan discursos con capacidad de sintesis para cada uno de los casos seleccionados. Las Comunidades Autónomas cuentan con estructuras y competencias territoriales para coordinar y marcar directrices en torno a la orientación y formación docente. No obstante, las soluciones estructurales dadas en cada caso son diferentes. Se encuentran desde diseños más centralizados como en LR, BAL, MAD, CyL o MUR. Hasta modelos más descentralizados presentes en AND con su red de unidades provinciales, en el PV con los Berrizagunes ${ }^{8}$, en NAV con el CREENA ${ }^{9}$,

7 - Disponible en: <https://www.gifex.com/fullsize/2009-12-08-11364/Mapa-de-las-Comunidades-Autnomas-de-Espaa.html>. Acceso en: 01 agt. 2018.

8- Los Berritzegunes se establecen como Servicios de Apoyo de carácter zonal y/o territorial para la innovación y mejora de la educación en los niveles de enseñanza no universitaria en la Comunidad Autónoma del País Vasco.

9- Centro de Recursos de Educación Especial de Navarra (CREENA). Es un centro especializado de apoyo al sistema educativo, que contribuyen a la mejor integración escolar del alumnado en el sistema educativo en la Comunidad Autónoma de Navarra. 
o en CAN donde se lleva a cabo una orientación colaborativa entre etapas. Finalmente, el modelo más descentralizado se encuentra en VAL, que otorga una gran capacidad de decisión al centro educativo.

La existencia de estas estructuras de coordinación permite detectar discursos con la propiedad de ofrecer una visión general de la orientación en cada territorio. En consecuencia, se pueden seleccionar prototipos discursivos representativos de especialistas, en puestos de coordinación autonómica, que permiten vincular su percepción del mercado de trabajo vinculado a la Orientación Académica y Profesional al contexto socioeducativo (ver cuadro 3). Dados los altos costes y la dificultad de acceso se han realizado diez entrevistas, que aunque podrían ser más, la peculiaridad de representar discursos con capacidad de síntesis garantiza un nivel de saturación mínimo, pero suficiente, de la información.

Cuadro 3- Composición de la muestra por casos de estudio y código de entrevista

\begin{tabular}{|c|c|c|}
\hline CCAA & Técnico & Cód. \\
\hline AND & Técnico Servicio de Orientación Educativa y Atención a la Diversidad. & 01anda \\
\hline BAL & Técnico de Orientación Servei de Soporte Educatiu. & 02bale \\
\hline CAN & $\begin{array}{l}\text { Responsable Área de Necesidades Específicas de Apoyo Educativo y Orientación Educativa y } \\
\text { Profesional, Innovación, y Promoción Educativa. }\end{array}$ & 03cana \\
\hline CYL & $\begin{array}{l}\text { Servicio de Atención al Alumnado con Necesidad Especifica de Apoyo Educativo, Orientación y } \\
\text { Convivencia, Dirección General de Innovación Educativa y Formación del Profesorado. }\end{array}$ & 04cyl \\
\hline $\mathrm{LR}$ & Coordinación Servicio de Orientación Educativa y Atención a la Diversidad. & 10rio \\
\hline MAD & Técnico de coordinación, Consejería de Educación. & 05madr \\
\hline MUR & Responsable coordinación Dirección General de Ordenación Académica y Atención a la Diversidad. & 06murc \\
\hline NAV & Responsable coordinación Servicio de Atención a la Diversidad y Necesidades Especiales. & 07nava \\
\hline PV & Técnico Berritzegune Central. & 08pv \\
\hline VAL & Responsable coordinación Servicio de Ordenación Académica. & 09vale \\
\hline
\end{tabular}

Fuente: Protocolo de investigación.

\section{Técnica}

La técnica aplicada ha consistido en la entrevista orientada de manera inducida. Mediante este procedimiento se pretende captar las características específicas de los discursos de responsabilidades arquetípicas y los contextos de motivación de su producción. Esta técnica tiene como ventaja la posibilidad de conseguir una mayor interacción, que permite profundizar más en la motivación personal frente a un problema social (BALLESTER, 2006). Interesa por tanto acceder al discurso en sí, para categorizar en base a los factores discursivos que aparecen. El guión elaborado para substraer la visión de los entrevistados se ha concretado en una batería de preguntas conectadas con los objetivos del estudio, a saber:

- La Educación Secundaria Obligatoria (ESO) es una etapa en la que se comienza a detectar el abandono educativo temprano: ¿en qué medida puede influir esto en el proceso de orientación? 
- Las tasas de paro, actividad y ocupación varían entre las comunidades autónomas. Teniendo en cuenta el escenario social y económico de su comunidad autónoma, y pensando en la labor orientadora del profesorado de la ESO ¿tienen margen en el aula estrategias de orientación que prevengan el desempleo juvenil?

- Es conocido que el abandono escolar temprano es una acción que la Unión Europea, y España están empeñadas en reducir de forma considerada. Concretamente, ¿qué medidas considera usted que deberían de tomarse para corregir ese abandono escolar temprano que parece incidir de alguna manera en la inserción profesional de estos jóvenes que no terminan la enseñanza obligatoria?

- Aunque ya hemos hablado de algunos factores que influyen en la orientación, a modo de resumen, ¿qué importancia considera que tienen en su comunidad autónoma: el fracaso escolar, las demandas y la inserción en el mercado laboral y la oferta educativa en la CCAA, tanto de Formación Profesional como de Bachillerato y las posibles carreras universitarias?

\section{Estrategia Analítica}

El estilo analítico adoptado se centra en los casos. En primer lugar, se hace hincapié en la exposición e interpretación de los relatos caso a caso, pero siguiendo una lógica de análisis comparativo dentro de cada perfil o within case analysis. En segundo lugar, se aborda un análisis cruzado de casos pertenecientes a distintos perfiles o cross-case analysis (MILES; HUBERMAN, 1984). Ambas fases permiten construir una descripción tipológica (WEISS, 1994) y analizar la complejidad de los casos reales.

La técnica de análisis se fundamenta en el Método Comparativo Constante, pues permite descubrir conceptos y relaciones y organizarlos en esquemas teóricos explicativos (OSSES; SÁNCHEZ; IBAÑEZ, 2006). Particularmente, se adopta un proceso deductivo-inductivo, partiendo de categorías iniciales para proceder a extraer categorías emergentes a partir de los textos seleccionados (OSSES; SÁNCHEZ; IBAÑEZ, 2006).

\section{Categorías Iniciales}

Según el modelo teórico adoptado, se observa que la Orientación Académica y Profesional se asocia a diferentes elementos para coadyuvar el estrechamiento entre mercado de trabajo y centro educativo. Por un lado, con relación al conocimiento del entorno se debe de orientar académicamente teniendo en cuenta las transiciones de etapas y niveles y las transiciones entre centro educativo y ocupación. Y, por otro, para orientar profesionalmente hay que tener presente aspectos relativos a la ocupación, los procesos de inserción laboral y la elección vocacional. A partir de estas premisas se diseñan dos categorías teóricas para iniciar el análisis de la percepción del mercado de trabajo. El programa informático utilizado es ATLAS.ti 6.1:

- ORIENTACIÓN ACADÉMICA_F: = Abandono | itinerarios | transición| Orient* | temprano| educativa| académica| secundaria| universidad| prematuro| 
- ORIENTACIÓN VOCACIONAL_F: = Vocación | desempleo | paro| Orient* | mercado| profe* paro| Orient* | construcción| periodismol ingenieríal informátical trabajo| empleo| laboral|

\section{Análisis}

A continuación, se aborda el análisis de las entrevistas, para ello se ha dividido la presentación en dos apartados. El primero se dedica a la presentación del análisis caso a caso dentro de cada perfil socioeducativo. Y el segundo a presentar los resultados del estudio comparativo de casos entre perfiles socioeducativos. A su vez, cada uno de ellos se subdivide en dos sub-apartados más según la categoría inicial o categoría emergente que se aborde.

\section{Análisis caso a caso dentro de cada perfil socioeducativo}

\section{Categoría inicial Orientación Académica}

En este sub-apartado se muestra la descripción de cada discurso dentro de cada perfil, haciendo hincapié en los criterios de búsqueda incluidos en la categoría teórica inicial Orientación académica.

Perfil 1: tasa paro juvenil (TPJ) alta, tasa abandono educativo temprano (TAET) alta y perfil actividad económica (PAE): AND (p), BAL(s), VAL (i), CAN(s) y MUR (p) ${ }^{10}$

En los discursos de los técnicos de Andalucía, Murcia y Valencia reconocen al mercado de trabajo la suficiente influencia para condicionar la toma de decisiones del alumnado en sus transiciones (ver cuadro 4). En otras palabras, le confieren un poder de atracción relevante sobre los estudiantes, especialmente en momentos de abundante oferta laboral de baja cualificación. Por ejemplo, en el discurso del técnico de Valencia se indica que se ha dado un determinado perfil de estudiantes que, seducidos por los salarios, decidió abandonar las aulas cuando su lugar era el centro educativo. Incluso, en el discurso de Murcia se reconoce un grado mayor de influencia, pues se le percibe como un imán que atrae hacia él incluso a los críos normales seducidos por los salarios.

A esta cualidad para extraer recursos humanos de los centros educativos, cuando se entiende que se ha producido de un modo prematuro, se le otorga una fuerte negatividad. Buena prueba de ello queda ilustrada en los apelativos otorgados a los estudiantes que cambiaron las aulas por el trabajo antes de tiempo como, por ejemplo, la generación de los perdidos o de los vulnerables, cuya decisión consideran que truncó sus trayectorias formativas, desdibujando su horizonte profesional.

10 - (p) Sector de actividad económica Primaria; (i) Sector de actividad económica Industrial; (s) Sector de actividad económica Servicios. 
Cuadro 4- Reproducción de los registros de las entrevistas para la categoría Orientación Académica. Casos AND, MUR y VAL

\section{P 3: ENTREVISTA 2 ANDALUCÍA.txt - 3:11 (58:58)}

... pero sí hay chavales que han abandonado, y han abandonado sobre todo con el boom de la construcción. Y cuando abandonaron con el boom de la construcción hay muchos que ahora no saben qué hacer.

\section{P 9: ENTREVISTA 8 MURCIA 7-5-2015.txt - 9:28 (104:104)}

Esa situación de bonanza económica que hubo aquí, que provocó que generaciones de alumnos, de críos normales que optaron por el trabajo fácil en un momento determinado y con un sueldo relativamente alto, pues ahora mismo tenemos una generación muy, muy joven en el paro y parados con muy malas perspectivas. Con lo cual eso, evidentemente claro que marca...Aquí, una de las cosas que se les da en Murcia y en el levante en general, que ha dependido mucho de la construcción durante unos años, y por eso tenemos las tasas de abandono educativo temprano que tenemos, supuso que una generación o varias generaciones de jóvenes no estudiaran porque había muchas oportunidades laborales.

\section{P 2: ENTREVISTA 10 VALENCIA 21-5-2015.txt - 2:3 (19:20)}

Sí, tiene que ver, además de lo que ya he comentado, hay que tener en cuenta que hemos tenido unas décadas que por el tipo de economía que hemos tenido y el tipo de mercado laboral, realmente ha sido muy complicado a determinados perfiles de alumnos convencerles de que su sitio estaba en la escuela, en la formación, en los institutos, cuando se podía tener un buen sueldo con un trabajo para el que se requería poca cualificación relacionado, por ejemplo, con el mundo de la construcción.

* Corresponde al código que asigna el programa de análisis cualitativo ATLAS-ti, a los fragmentos seleccionados de las transcripciones de las entrevistas realizadas.

Fuente: Protocolo de investigación.

En los discursos de los técnicos de Baleares y de Canarias (ver cuadro 5) también se confiere al mercado de trabajo la capacidad de provocar transiciones antes de tiempo, y como en los tres casos anteriores, dicha influencia se valora negativamente. Por ejemplo, en Baleares se reconoce la capacidad de atracción del mercado sobre un determinado alumnado, caracterizado por su mayor interés o necesidad por obtener ingresos. Debido a ello, se percibe la orientación como una lucha contra la persuasión de los salarios. Por su parte, en el discurso de Canarias yendo más allá, enfocando su discurso en una visión más estructural que circunstancial, reconocen que orientar teniendo en cuenta las condiciones del mercado de trabajo local puede desesperanzar a los estudiantes.

Cuadro 5- Reproducción de los registros de las entrevistas para la categoría Orientación Académica. Casos BAL y CAN

\section{P 7: 6 BAL 17-3-2015.txt - 7:14 (78:78)}

No, no, claro, es que es real, que esos alumnos abandonan... Pues había muchos niños de diferentes culturas y esos veías claramente que no iban a continuar, que no podían continuar. Y para lo que es el departamento de orientación, para el orientador, para los integrantes del departamento de orientación era una lucha constante. 0 sea, es el tema económico, que es real, es muy real.

P 4: 3 CAN 10-3-2015.txt - 4:8 (30:30)

Yo creo que es complicado, es decir, se tiene margen siempre que sigas determinado itinerario al marcar determinadas actuaciones... El que ellos exploren la realidad de cómo está y muchas veces pues es realmente difícil que... Y más en Canarias y en determinadas zonas, el poder tener una actuación que no les lleve a la desesperanza por así decirlo, ¿no?, de las actuaciones.

Fuente: Protocolo de investigación. 
Perfil 2: TPJ y TAET menos altas, PAE: CyL (i), LR (i), MAD(s)

Los casos incluidos en el perfil 2 muestran convergencias entre ellos (ver cuadro 6). En el caso concreto del técnico de Castilla y León reconoce que la abundante oferta de trabajos de baja cualificación influyó para que se produjesen transiciones prematuras del aula al puesto de trabajo. Y, al igual que los técnicos del perfil 1, connota negativamente la capacidad extractora del mercado de trabajo. Además, siguiendo un patrón similar al observado describe como generación perdida a aquellos jóvenes que optaron por el trabajo, abandonando las aulas antes de concluir una adecuada formación.

El discurso del técnico de Madrid se aleja de todos los anteriores. Sin dejar de reconocer la importancia del mercado de trabajo, no le otorga una connotación negativa al mismo. Particularmente, entiende que el modelo adecuado de conectar las habilidades laborales con la formación es mediante una adecuada orientación profesional, que permita optimizar la toma de decisiones por parte del alumnado.

En consonancia con el cambio de percepción apuntado en el discurso de Madrid, en el discurso de La Rioja se encuentra la connotación más positiva vinculada a la relación entre sistema educativo y mercado de trabajo. Una prueba de ello queda patente en que no se alude a una generación perdida, ni desorientada con relación al abandono educativo temprano, sino que se describe a unos jóvenes que toman decisiones y transitan del aula a la ocupación acorde con sus decisiones y las oportunidades laborales del momento.

Cuadro 6- Reproducción de los registros de las entrevistas para la categoría Orientación Académica. Casos: CyL, MAD y LR

\footnotetext{
P4: 04cyl 17-3-2015.txt - 7:24

Pero no nos quedamos aquí, creemos firmemente que también hay que atender a ese alumnado que ya cuenta como abandono escolar temprano, no podemos dejar una "generación perdida".

P6: 5 MAD 16-3- 2015.txt - 6:18 (90:90)

En un momento en que las tasas de desempleo son importantes el abandono no es una solución, la solución es seguir formándose y mejorando en determinados campos. Si para esto nosotros recogemos información sobre lo que las estadísticas nos dan desde instituciones, pues nos dicen cuáles son los niveles de inserción según familias profesionales, también la Comunidad de Madrid ofrece de los titulados de formación profesional qué nivel de iniciación, qué perfiles son los más demandados, los menos demandados.
}

P5: 4 LR 12-3-2015.txt - 5:12 (59:59)

Yo lo que te voy a plantear es de que si el abandono escolar realmente se ha producido es porque hay alternativa realmente a ese abandono Vamos a ver, yo creo que en estos momentos el mundo laboral que todos teníamos ahí, por lo menos en esta comunidad autónoma era una salida del abandono escolar porque el crío que cumplía 16 años tenía puesto de trabajo. Y así somos unos beneficiados en eso, porque es una comunidad autónoma quizá distinta de los demás, pero la realidad es que hoy eso no existe.

Fuente: Protocolo de investigación.

Perfil 3: TPJ alto, TAET menos alto, PAE industrial: NAV (i) y PV (i)

En los discursos de Navarra y País Vasco (ver cuadro 7) se distancian aún más de los anteriores al no mencionar explícitamente al mercado de trabajo cuando se abordan 
asuntos como los itinerarios o el abandono educativo temprano. Es decir, no se evoca al mercado de trabajo como un referente manifiesto en el proceso de orientación, aunque se puede intuir que su influencia existe. Por este motivo se deduce que ellos entienden que las mejores garantías profesionales para el individuo pasan porque permanezca en el sistema educativo el mayor tiempo posible. No les preocupa tanto la capacidad de extracción de los recursos humanos de los centros educativos como aumentar la esperanza de vida escolar del alumnado. Para ello prestan atención, sobre todo, en las transiciones entre la formación obligatoria y los primeros ciclos de la post-obligatoria, especialmente, en aquellos perfiles con tendencia a fracasar escolarmente.

Cuadro 7- Reproducción de los registros de las entrevistas para la categoría Orientación Académica. Casos: NAV y PV

\footnotetext{
P 8: 7 NAV.txt - 8:9 (54:54)

Nosotros lo que intentamos es que el alumno lo tengamos escolarizado el mayor tiempo posible, pero dándole una respuesta lo más adecuada a lo que necesita. De ahí que vengan todas estas orientaciones que podemos tener a la diversificación, a los distintos caminos que puede seguir en $4^{\circ}$ de la ESO, a la Formación Profesional Básica, a los itinerarios que podamos tener o programas de talleres profesionales que tenemos también.

P 1: 1 PV 5-3-2015.txt - 1:15 (103:104)

0 sea, no sé. Ya te digo el porcentaje no es alto... Más preocupante es eso, el que termina en un grado medio e igual ahora mismo no encuentra trabajo aunque parece que empieza un poquito la cosa a moverse... pues no encuentran trabajo y hacen todo el grado medio. No sé, parece que la Formación Profesional... Pero el porcentaje de jóvenes que terminan, que no sacan el graduado en su momento, ya te digo que por aquí es bastante bajo, tienen distintas opciones.
}

Fuente: Protocolo de investigación.

\section{Categoria Orientación Profesional}

Perfil 1: TPJ: alta, TAET: alta, PAE: AND (i), BAL(s), VAL (i), CAN (s) y MUR (p)

Bajo esta categoría, los técnicos de Andalucía, Murcia y Valencia (ver cuadro 8) muestran de nuevo una visión negativa del mercado de trabajo como receptor de egresados, dada las limitaciones del entorno como referente vocacional y al dominio de la influencia del mercado sobre el sistema educativo. Por ejemplo, en el discurso del técnico de Andalucía se reconoce que, ante una realidad tan adversa como la dibujada por las altas tasas de desempleo juvenil, no se orienta hacia la profesión, sino hacia la empleabilidad. Aunque manifiestan que ofrecen orientación laboral, subrayan que conocer las opciones del mercado en sí mismo no capacita, que es el objetivo perseguido. Una percepción similar se recoge en el discurso del técnico de Murcia, que resalta la impotencia que genera orientar profesionalmente ante un mercado de trabajo yermo en oportunidades para los jóvenes. Por su parte, en el discurso del técnico valenciano también se tiene presente la orientación laboral, pero dadas las condiciones adversas del mismo, insiste en que la orientación vocacional debe de perseguir preparar para la vida y no para la ocupación.

Aún con sus matices, los tres técnicos convergen a la hora de resaltar las dificultades que plantea apoyarse en un mercado de trabajo para ayudar en la elección vocacional. En 
coherencia con esta visión, los técnicos de Andalucía y Valencia conciben la orientación vocacional como una herramienta para ayudar a los alumnos a trascender a una realidad laboral circundante adversa. En el de Murcia como un esfuerzo baldío ante la ausencia de oferta de trabajo. En este sentido, de nuevo coinciden en ofrecer una visión negativa de la relación entre sistema educativo y mercado de trabajo.

Cuadro 8- Reproducción de los registros de las entrevistas para la categoría Orientación Profesional. Casos: AND, MUR y VAL

\begin{abstract}
P 3: 2 AND.txt - 3:22 (80:80)
Las tasas de desempleo son brutales, las tasas de desempleo juvenil son inaceptables y nosotros tenemos que hacer que las personas tengan, no ya una empleabilidad como te he dicho, sino el desarrollo de un proyecto vital que incluye un empleo. 0 sea, yo no hago orientación para formar a un trabajador y hago orientación para formar a una persona que tendrá un empleo. Y ese empleo no será el que condicione lo que yo haga y tenga que desarrollar una serie de capacidades que no necesariamente van a ser útiles para una sola actividad profesional, sino que yo tendré que desarrollar una serie de capacidades que me formarán como persona y con esa formación puedo acceder a una.
\end{abstract}

P 9: MUR 8 7-5-2015.txt - 9:20 (78:78)

Hombre, ahí la información es muy importante. Que tengan información de todas las posibilidades y todas las opciones académicas que tienen delante de ellos es muy importante. Pero si no hay oportunidad laboral, por mucha información y por mucha orientación que les demos difícilmente van a trabajar.

\title{
P 2: 10 VAL 21-5-2015.txt - 2:3 (19:19)
}

Bien, a nivel general el mercado laboral ha cambiado, creo yo. En el sentido en que no se busca el mismo personal cualificado de hace unos años a ahora. Actualmente las empresas buscan más personas competentes. Y ese nivel, ese nivel no solamente afecta la evolución del mercado laboral, no solamente afectan a la orientación, es que acaba afectando a toda la educación. Porque preparamos, no para incorporarse al mundo del trabajo, pero sí para convivir en una sociedad y en nuestra sociedad está el mundo del trabajo.

Fuente: Protocolo de investigación.

De un modo parecido a los tres anteriores, en los discursos de las Islas Baleares y Canarias (ver cuadro 9) se percibe una relación entre el sistema educativo y el mercado de trabajo dominada por las condiciones del segundo. La capacidad del mercado para extraer los recursos humanos de las aulas en periodo expansivo, como la frustración que genera la ausencia de oferta laboral en periodo de crisis convierte al mercado de trabajo en un referente vocacional no preferente. Al igual que se ha recogido en discursos anteriores, esta relación determina cómo se percibe la Orientación Profesional. Por ejemplo, en el caso del técnico de Baleares se siente la orientación como una lucha para contrarrestar la capacidad de atracción de las ofertas de trabajo de baja cualificación. Mientras que el técnico de Canarias manifiesta que se evita su referencia como inspirador de vocaciones. Ambas visiones reflejan una imagen negativa del mercado de trabajo.

Cuadro 9- Reproducción de los registros de las entrevistas para la categoría Orientación Profesional. Casos: CAN

\footnotetext{
P 4: CAN 10-3-2015.txt - 4:14 (80:80)

Es un factor cuando tiene que ver con los centros de Formación Profesional, ese sí que es un factor o cuando se está trabajando este tipo de itinerarios. Pero sinceramente la política de orientación que se marca en casi todos los sitios es no dejarse influir en la toma de decisiones exclusivamente por lo que es el mercado laboral o lo que es el propio sector, porque si no el alumnado sería incapaz de emprender cualquier tipo de movilidad para situarse en el mercado laboral.
}

Fuente: Protocolo de investigación. 
Perfil 2: TPJ y TAET menos altas, PAE: CyL (i), LR (i) y MAD (s).

En los discursos de los técnicos de Madrid y La Rioja (ver cuadro 10) se reconoce la influencia de la oferta y la demanda laboral sobre la elección vocacional. En esta ocasión no se percibe al mercado de trabajo como un elemento al que trascender, sino al que afrontar teniendo en cuenta sus peculiaridades. En otras palabras, se reconoce como un factor que genera incertidumbre, que es difícil de escrutar, pero que ofrece oportunidades. Particularmente, el técnico de La Rioja ofrece de nuevo la connotación más positiva del mercado de trabajo, pues no se le otorga la capacidad de ejercer una influencia negativa sobre el alumnado. Más bien lo muestra como una opción legítima a tener en cuenta en el proyecto vocacional.

Además, aparece una convergencia entre estos dos casos que es interesante destacar. Esta coincidencia estriba en que cuando se evoca las dificultades del mercado de trabajo se aluden a estudios que conducen a profesiones como las humanidades los informáticos o de periodismo, dejando entrever que uno de los escoyos de la orientación profesional reside más en la incertidumbre que generan las fluctuaciones de la oferta y la demanda de ocupaciones, que en la capacidad de extracción prematura de los recursos humanos del mercado laboral. Finalmente, en el discurso del técnico de Castilla y León se esgrime un conjunto de medidas cuyo denominador común consiste en conectar mejor el sistema educativo con el mercado de trabajo, de lo que se deduce la necesidad de mejorar el encaje entre ambos. No obstante, no aparece el mercado de trabajo como un referente vocacional manifiesto.

Cuadro 10- Reproducción de los registros de las entrevistas para la categoría Orientación Profesional. Casos: CyL, LR y MAD

P4: 04 CYL 17-3-2015.txt - 8:24

Combatir el paro juvenil y mejorar las tasas de empleo es una responsabilidad de todas las administraciones. Desde la Consejería de Educación trabajamos en varias líneas educativas: Orientación en Secundaria, Bachillerato, Formación Profesional y Centros de Adultos. Pruebas para obtener acceso a ciclos de Formación Profesional y para la obtención del Título de Graduado en Educación Secundaria. Unión de las empresas con el sistema educativo; Formación Profesional Dual, etc. Asignaturas de emprendimiento para el alumnado desde $3^{\circ} 04^{\circ}$ de la Educación Secundaria Obligatoria.

P5: 4 LR 12-3-2015.txt - 5:27 (127:127)

Hombre, lo que sí influye desde luego, es que si estudias clásicas pues tú sabes que el futuro ahora es magnífico, pero que hace diez años era más negro que Machín. Y yo creo que eso los orientadores lo dejan claro, además. Que además de orientar, pues muy bien, pero cuidado. En la orientación también está el comer.

P6: ENTREVISTA 5 MADRID 16 DE MARZO DE 2015.txt - 6:20 (112:112)

Pues sí, sí, sí, claro que hay, hay movimiento que el propio mercado surge. Hubo una época donde la informática era el no va más, todo era informática. Había un momento que lo que se necesitaban eran, a raíz incluso de una serie de televisión, creo que también se habló de periodistas. Todo, desde los modelos sociales y los medios de comunicación ponen al alcance pues despiertan y desarrollan.

Fuente: Protocolo de investigación.

Perfil 3: TPJ alto, TAET menos alto, PAE: NAV (i) y PV (i)

En los discursos de los técnicos de Navarra y País Vasco (ver cuadro 11) también se reconoce la influencia de la oferta y la demanda laboral sobre la elección vocacional. Además, en ellos aparece de nuevo la convergencia advertida anteriormente. Al igual que 
en los discursos de Madrid o La Rioja se mencionan profesiones que requieren estudios superiores, en estos dos casos a la de ingenieros, cuando se centran en las dificultades encontradas a la hora de orientar vocacionalmente. De nuevo, se percibe que la dificultad reside en la incertidumbre que generan las fluctuaciones en la oferta y la demanda de determinadas profesiones. En consonancia con esta visión, no se percibe al mercado de trabajo como un elemento al que trascender, sino al que afrontar teniendo en cuenta sus peculiaridades. En estos discursos se reconoce una relación entre centro educativo y mercado de trabajo donde gana el pulso el primero. La clave es que en estos discursos se vislumbra que han conseguido encauzar a los estudiantes hacia itinerarios formativos adaptados, impidiendo aumento de transiciones prematuras vinculadas a la atracción de las demandas laborales de baja cualificación.

Cuadro 11- Reproducción de los registros de las entrevistas para la categoría Orientación Profesional. Casos: NAV y PV

P 8: 7 NAV 18 0-3- 2015.txt - 8:20 (114:114)

Pues en parte sí pues porque yo estoy pensando ahora mismo que hasta hace cinco años las ingenierías pues tenían una demanda terrible, y ahora en lo que se está dando una demanda grandísimas es con el Magisterio. Y sí, yo creo que hay modas... Por ese lado así en general no (desempleo), porque ahí el problema que tenemos o en cuanto detectamos que hay un alumno que está o desencantado del sistema educativo o que su rendimiento se ve que no va a llegar a alcanzar la titulación en la Educación Secundaria Obligatoria, pues intentamos buscar alternativas.

P 1: 1 PV 5-3-2015.txt - 1:14 (104:104)

Porque es que han visto de todo. Hace poco las ingenierías eran bufffff y luego están viendo que todos los ingenieros están buscándose la vida en otros sitios, aunque sea coyuntural. Entonces, bueno, pues es un factor cambiante cómo está el mercado laboral. El tema es que es tan peliagudo que bueno, cuando eres orientadora en el centro, te suelen llegar alumnado y te dicen: “¿yo qué elijo para el año que viene?, quiero una carrera con salidas". Es que es algo tan cambiante. Yo no soy adivina y no sé dentro de cinco años cómo estará el panorama, no tengo ni idea. Que es el tiempo que tardan en terminar los estudios. ¿Eso hasta qué punto...? A ver, si tiene en cuenta cuando el alumnado está tratando de elegir al final de etapa, sobre todo, al final de bachillerato

Fuente: Protocolo de investigación.

\section{Análisis comparativo de casos entre perfiles socioeducativos}

\section{Categorías emergentes según el flujo de la relación}

De los casos estudiados, dos señalan las pautas polares: en un extremo (-) (-), la visión del mercado de trabajo se muestra muy negativa tanto como por su capacidad para truncar carreras académicas prematuramente, como de contexto al que hay que trascender por sus ausencias oportunidades laborales. En el extremo opuesto $(+)(+)$, reúne a aquel que sostiene que el mercado de trabajo es una referencia permanente en la orientación vocacional a pesar de las fluctuaciones de la oferta y la demanda de las ocupaciones.

$$
\text { MUR (-) (-) ------------- LR (+) (+) }
$$

En esta línea polar se sitúan los demás casos. Su posición relativa, más próxima a cada uno de los extremos, viene determinada por su percepción del mercado de trabajo en relación con el sistema educativo y la función de orientación. Son casos que encierran 
mayor complejidad que los supuestos polares y cuyo escalamiento resulta difícil, en consecuencia es necesario concretar aún más los criterios para su clasificación.

Los casos intermedios plantean la necesidad de especificar categorías que ayuden a la clasificación de los mismos. En este sentido, el análisis, aparte de detectar convergencias, ha permitido generar categorías emergentes vinculadas al objeto de estudio que facilitan la comparación.

A partir de las categorías de análisis iniciales de Orientación Académica que registraba entre otros factores el abandono educativo temprano y de Orientación Vocacional, que se vinculaba especialmente a las tasas de desempleo, se han detectado dos flujos en la relación entre centro educativo y el mercado de trabajo. La concreción del sentido de la relación ha permitido concretar dos categorías emergentes que hemos denominado: capacidad extractora de recursos prematura y receptor de egresados a término (ver gráfico 2).

Gráfico 2- Flujos en la relación mercado laboral y centro educativo

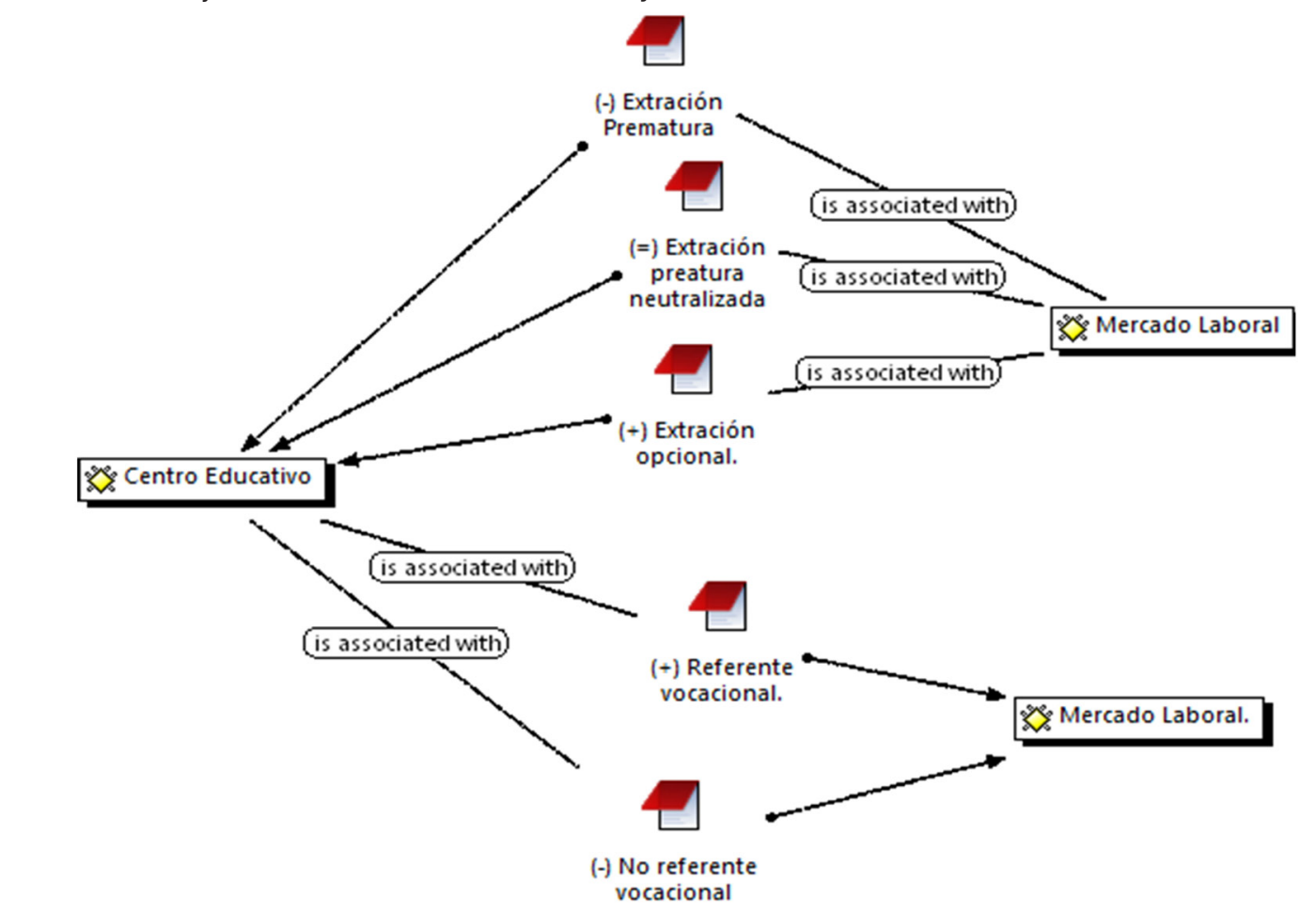

Fuente: Protocolo de investigación.

Por lo que respecta a la capacidad extractora de recursos humanos prematura, alude a la capacidad percibida del mercado de atraer al alumnado una vez cumplidos los 16 años, sin haber obtenido el título de Enseñanza Secundaria Obligatoria o no continuar con estudios Superiores. En esta categoría un símbolo negativo (-) significa que se reconoce 
esta influencia como negativa; un símbolo (=) significa que se ha contrarrestado esta la capacidad extractora y (+) que se reconoce como una opción legítima que el estudiante decida abandonar el aula por un puesto de trabajo a esta edad.

Por su parte, la categoría receptor de egresados a término permite tener en cuenta la percepción del mercado de trabajo como elemento de referencia en la elección vocacional de un individuo al que ya se considera una trayectoria educativa suficiente. Es decir, que no ha abandonado, a su juicio, prematuramente sus itinerarios formativos. En esta categoría un símbolo negativo (-) significa que hay que trascender al mercado como referencia en la ayuda a la elección vocacional y (+) que en la función de orientación vocacional se percibe el mercado de trabajo como una meta o referencia, a pesar de sus incertidumbres.

Una vez determinadas las categorías, se cruzan ambas y sus propiedades (ver cuadro 12). La distribución permite estructurar mejor los discursos acordes con su visión del mercado de trabajo. Como se observa, los discursos del perfil 1 se diferencian del resto por la doble negatividad que otorgan al mercado de trabajo tanto en su capacidad extractora como receptora de egresados. Frente a ellos se sitúan los discursos del perfil 2 donde se observa una valoración positiva en ambas categorías. Finalmente, la divergencia de los discursos del perfil 3 con el resto estriba en que hacen hincapié en la idea de que se ha contrarrestado o neutralizado la capacidad extractora de los recursos humanos prematuramente del mercado de trabajo.

Cuadro 12- Percepción del Mercado Laboral desde la función de Orientación Académica y Profesional por casos de estudio

\begin{tabular}{|c|c|c|c|c|}
\hline & \multicolumn{4}{|c|}{ Capacidad Extractora } \\
\hline \multirow{4}{*}{$\begin{array}{l}\text { Receptor } \\
\text { egresados }\end{array}$} & & - & $=$ & + \\
\hline & + & & $\begin{array}{l}\text { NAV (p3) } \\
\text { PV (p3) }\end{array}$ & $\begin{array}{c}\mathrm{LR}(\mathrm{p} 2) \\
\operatorname{MAD}(\mathrm{p} 2)\end{array}$ \\
\hline & $=$ & CyL (p2)* & & \\
\hline & - & $\begin{array}{l}\text { AND (p1) } \\
\text { MUR (p1) } \\
\text { BAL (p1) } \\
\text { CAN (p1) } \\
\text { VAL (p1) }\end{array}$ & & \\
\hline
\end{tabular}

Nota: (p1) perfil 1, (p2) perfil 2, (p3s) perfil3. *La información obtenida impide una clasificación más ajustada.

Fuente: Protocolo de investigación.

\section{Categorías emergentes según propiedades de la relación}

No obstante, se ha observado que según se connota al mercado de trabajo, se vinculan un conjunto de propiedades a la función de Orientación Académica y Profesional. Esta peculiaridad ha permitido extraer dos categorías emergentes más que han coadyuvado la comparación entre perfiles, a saber: Equilibrio de la Relación y Asesoramiento Vocacional. 
La categoría Equilibrio de la Relación recoge cómo se percibe el balance entre mercado de trabajo y sistema educativo. La relación se decanta del lado del primero cuando la influencia del mercado impide la consecución de los objetivos formativos, bien porque trunca carreras académicas, bien porque no absorbe a la población de egresados. En estos casos se observa que existe una relación dominada por el mercado de trabajo, donde las transiciones prematuras se producen porque la atracción de los salarios se superpone a la búsqueda vocacional a través de itinerarios académicos. Al igual que la ausencia de oportunidades impide criterios claros de asesoramiento vocacional. También puede escorarse una relación decantada hacia el lado del sistema educativo cuando, desde los centros, se percibe que se ha contrarrestado la influencia del mercado de trabajo para extraer los recursos humanos prematuramente y consiguen mantener la búsqueda vocacional transitando aún por el sistema educativo. Finalmente, el tercer tipo de balance refleja un equilibrio (fluidez) entre ambos entornos, donde percibe la idea que el individuo transita del centro al mercado de trabajo siguiendo un patrón vocacional acorde con las reglas de la oferta y demanda laboral.

La categoría Asesoramiento Vocacional muestra una concepción de la Orientación Académica y Profesional según la imagen del mercado de trabajo percibida. Por un lado, en el discurso de AND el asesoramiento se percibe como una responsabilidad sobrevenida; como una actividad infructuosa en MUR; una lucha contra la influencia del mercado en BAL, un asesoramiento desesperanzador dada las tasas de desempleo en CAN. A esta concepción se la ha denominado Orientación Hostil. No quiere decir que no se tenga en cuenta al mercado de trabajo en ningún momento, sino que dadas sus circunstancias contextuales, como las altas tasas de desempleo juvenil, desde la orientación se marca como objetivo trascenderlo o dejarlo relegado a un segundo plano como referente en la elección vocacional. Por otro, se ha percibido una orientación estratégica enfocada a reducir la incertidumbre en la toma de decisiones vocacionales, a pesar de las fluctuaciones del mercado de trabajo.

En el cuadro 13 se recoge la distribución de los casos según el cruce de estas dos categorías emergentes más el perfil socioeducativo. Se observan tres divergencias globales según el modo de concebir la relación entre centro educativo y mercado de trabajo a través de la orientación profesional. Dentro de la primera divergencia se vislumbran aquellos que muestran una relación desequilibrada entre sistema educativo y mercado de trabajo a favor del segundo, vinculada a una percepción de la Orientación Hostil. Estos discursos son propios de contextos con altas TAET y TDJ (perfil 1). La segunda divergencia reúne a aquellos casos que perciben la relación entre sistema educativo y mercado de trabajo de un modo desequilibrado, pero a favor del primero. La Orientación Académica y Profesional se concibe como una estrategia de planificación ante un entorno más favorable. Los discursos agrupados bajo esta divergencia se concentran en contextos con TAET y TDJ bajos (perfil 3). Finalmente, la tercera divergencia se distancia de las otras porque muestra una relación fluida entre mercado y sistema educativo, vinculada a una orientación de tipo estratégica. Este discurso es propio de contextos con TAET medias, pero bajas TDJ (perfil 2). 
Cuadro 13- Distribución de los casos según equilibrio de la relación, asesoramiento vocacional y perfil socioeducativo

\begin{tabular}{|c|c|c|c|c|c|c|}
\hline \multirow{3}{*}{ Perfil } & \multirow{3}{*}{ Caso } & \multicolumn{3}{|c|}{ Equilibrio de Relación* } & \multicolumn{2}{|c|}{ Asesoramiento Vocacional } \\
\hline & & \multicolumn{2}{|c|}{ D } & \multirow[t]{2}{*}{$\mathrm{E}$} & \multirow{2}{*}{ Hostil } & \multirow{2}{*}{ Estratégica } \\
\hline & & SE & $\mathrm{ML}$ & & & \\
\hline \multirow{5}{*}{$\mathrm{P} 1$} & AND & & $x$ & & $x$ & \\
\hline & MUR & & $x$ & & $x$ & \\
\hline & BAL & & $x$ & & $x$ & \\
\hline & CAN & & $x$ & & $x$ & \\
\hline & VAL & & $x$ & & $x$ & \\
\hline \multirow{3}{*}{ P2 } & CYL & & $x$ & & & \\
\hline & $L R$ & & & $x$ & & $x$ \\
\hline & MAD & & & $x$ & & $x$ \\
\hline \multirow{2}{*}{ P3 } & NAV & $x$ & & & & $x$ \\
\hline & PV & $x$ & & & & $x$ \\
\hline
\end{tabular}

*Nota: D (desequilibrado): hacia SE (sistema educativo) o ML (mercado de trabajo). Equilibrio (E).

Fuente: Protocolo de investigación.

\section{Conclusiones}

A la luz de la información analizada se han alcanzado algunos resultados relevantes. Cabe decir que aunque la muestra de casos podría ser mayor, y dada su amplitud, se esperaba una mayor casuística. Las claras convergencias encontradas permiten teorizar y plantear hipótesis con una validez sustentada en el procedimiento metodológico. Por ejemplo, se puede conjeturar que existen tres percepciones del mercado de trabajo extraídas de la relación de éste con el sistema educativo según contexto socioeducativo y que están influyendo en el proceso de Orientación Académica y Profesional.

En la primera, se agrupan los casos cuya imagen es muy negativa propia de contextos con altas TAET y de TDJ. En estos discursos se coparte la visión de un mercado de trabajo como una realidad que supera a la función de orientación vocacional, pues las lógicas del sistema educativo como laboral se encuentran disociadas. Además, aquí, es unánime considerar esta función como un ejercicio hostil. En la segunda, se agrupan los discursos del perfil 2 que otorgan una visión positiva al mercado propia de contextos con unas TAET no muy altas, pero con tasas de TDJ bajas. En consonancia con esta imagen, la ayuda vocacional se siente como un asesoramiento estratégico. Finalmente, la tercera, donde se agrupan todos los discursos del perfil 3, se percibe una visión neutral del mercado de trabajo, observado en los contextos con baja tasa de AET y TDJ.

También han quedado al descubierto los elementos que median en la relación entre sistema educativo y mercado de trabajo. Por un lado, se han detectado un flujo con dos sentidos, uno de extracción y otro de recepción. Por otro, se han extraído dos propiedades más una que alude al equilibrio de la relación entre centro educativo y entorno laboral y otra a la guía vocacional. 
No obstante, con la información que contamos no se puede confirmar dicha influencia, ni generalizar acordes a criterios cuantitativos, aunque sí ha permitido una aproximación a la comprensión de la Orientación Académica y Profesional por parte de agentes de responsabilidad de las Consejerías de Educación. Por estos motivos, las siguientes líneas de trabajo se centran en el profesorado de Educación Secundaria, con el fin de obtener mayor evidencia empírica. Para ello se pretenden desarrollar grupos de discusión y la administración de un cuestionario a una muestra significativa de centros educativos de toda España.

\section{Referencias}

ÁLVAREZ, Manuel. Orientación educativa: modelos, áreas, estrategias y recursos. Madrid: Wolters Kluwer Educación, 2012.

BALLESTER, Luis. El análisis semántico y pragmático de las entrevistas de investigación. Empiria, Madrid, n. 11, p. 107-129, ene./jun. 2006.

BEICHT, Ursula; WALDEN, Günter. How socially selective is the German system of initial vocational education and training? Transitions into initial vocational training and the influence of social background. Journal of Vocational Education \& Training, United Kingdom, v. 67, n. 2, p. 235-255, 2015.

BENAVENT, José Antonio. Desde la LGE de 1970 hasta la LOGSE de 1990. Valencia: Promolibro, 2002.

CEJUD0, Javier. Competencias profesionales y competencias emocionales en orientadores escolares. Revista de Currículum y Formación del Profesorado, Granada, v. 21, n. 3, p. 349-370, 2017.

CHISVERT, María José. Revisión del desarrollo de la orientación sociolaboral como política de empleo. Revista Española de Orientación y Psicopedagogía, Madrid, v. 25, n. 11, p. 8-24, 2014.

DE LA FUENTE, Raquel. Necesidades y recursos formativos de los tutores: paso previo en la elaboración de un Programa de Orientación y Tutoría para la Diversidad. Revista Española de Orientación y Psicopedagogía, Madrid, v. 21, n. 3, p. 571-586, 2010.

ECHEVERRÍA, Benito; MARTínEZ, Pilar. Luces entre sombras de la orientación. Revista Electrónica Interuniversitaria de Formación del Profesorado, Zaragoza, v. 18, n. 2, p. 1-13, 2015.

ESPAÑA. Ley 14/1970, de 4 de agosto, General de Educación y Financiamiento de la Reforma Educativa (LGE). Boletín Oficial del Estado, Madrid, n. 187, 6 agt. 1970, p. 12525-12546. Disponible en: <http:// www.boe.es/buscar/doc.php?id=BOE-A-1970-852>. Acceso en: 20 agt. 2017.

ESPAÑA. Ley Orgánica 1/1990, de 3 de octubre, de Ordenación General del Sistema Educativo. (LOGSE). Boletín Oficial del Estado, Madrid, n. 238, 4 oct. 1990, p. 28927-28942. Disponible en: <http://www.boe. es/buscar/doc.php?id=BOE-A-1990-24172> . Acceso en: 20 agt. 2017.

EURYDICE - Education, Audiovisual and Culture Executive Agency. Orientación académico-profesional en la educación obligatoria a tiempo completo en Europa. Madrid: Eurydice - Comisión Europea, 2012. 
ECHEVERRÍA, Benito; MARTíNEZ, Pilar. Luces entre sombras de la orientación. Revista Electrónica Interuniversitaria de Formación del Profesorado, Zaragoza, v. 18, n. 2, p. 1-13, 2015.

GONZÁLEZ, Viviana. La orientación profesional desde la perspectiva histórica cultural del desarrollo humano. Revista Cubana de Psicología, Habana, v. 20, n. 3, p. 260-268, 2003.

GRAÑERAS, Montserrat; PARRAS, Antonia (Coord.). Orientación educativa: fundamentos teóricos, modelos institucionales y nuevas perspectivas. Madrid: Ministerio de Educación - CIDE, 2008.

HOYT, Kenneth. A primer for career education. Washington, D.C: Department of Health, Education, and Welfare, 1977.

INSTITUTO NACIONAL DE ESTADÍSITCA. Encuesta Población Activa (EPA). Disponible en: <http://www. ine.es/dyngs/INEbase/es/operacion.htm?c=Estadistica_C\&cid=1254736176918\&menu=ultiDatos\&i $\mathrm{dp}=1254735976595>$. Acceso en: 25 sept. 2017.

KIM, Sung Won; BROWN, Kari-Elle; FONG, Vanessa. Credentialism and career aspirations: how urban Chinese youth chose high school and college majors. Comparative Education Review, Chicago, v. 60, n. 2, p. 271-292, 2016.

KNIGHT, Jasmine. Preparing elementary school counselors to promote career development recommendations for school counselor education programs. Journal of Career Development, Missouri, v. 42, n. 2, p. 75-85, 2015.

LORE, Van Praag et al. Programme content orientation in vocational education and training and life chances: a comparative study. Journal of Vocational Education \& Training, United Kingdom, v. 66, n. 3, p. 348-364, 2014.

MARTíNEZ, Pilar; PÉREZ Francisco Javier; MARTíNEZ, Mirian. Orientación profesional en educación secundaria. Revista Electrónica Interuniversitaria de Formación de Profesorado, Zaragoza, v. 17, n. 1, p. 57-71, 2014.

MILES, Matthew; HUBERMAN, Michael. Qualitative data analysis: a sourcebook of new methods. California: Sage, 1984.

MOLGAT, Marc; DESVHENAUX, Frédéric; LEBLANC, Patrice. Vocational education in Canada: do policy directions and youth trajectories always meet? Journal of Vocational Education \& Training, United Kingdom, v. 63, n 4, p. 505-524, 2011.

OLIVARES, María de los Ángeles; DE LEÓN Y HUERTAS, Carlota; GUTIÉRREZ, Pilar. El proceso de orientación profesional en los institutos de educación secundaria: el caso de Córdoba. Revista Española de Orientación y Psicopedagogía, Madrid, v. 21, n. 1, p. 81-92, 2010.

OBSERVATORIO DE LAS OCUPACIONES. Informe del mercado de trabajo de los jóvenes elaborado. Madrid: Servicio Público de Empleo Estatal. Ministerio de Empleo y Seguridad Social, 2016.

OSSES, Sonia; SÁNCHEZ, Ingrid; IBÁÑEZ, Flor. Investigación cualitativa en educación: hacia la generación de teoría a través del proceso analítico. Estudios Pedagógicos, Valdívia, v. 32, n. 1, p.119-133, 2006. 
SANTANA, Lidia; GARCÍA, Luis; CRUZ, Ana. El Programa de Orientación Educativa y Sociolaboral: un instrumento para facilitar la toma de decisiones en educación secundaria. Revista de Educación, Madrid, n. 351, p. 73-105, ene./abr. 2010.

SUPER, Donald; HALL, Douglas. Career development: exploration and planning. Annual Review of Pshychology, Palo Alto, v. 29, p. 333-372, 1978.

TIAN, Lin et al. Predictive validity of carrer decisión making profiles over time among Chinese college students. Journal of Career Development, United Kingdom, v. 41, n. 4, p. 282-300, 2014.

UNESCO. Replantear la educación: ¿hacia un bien común global? París: Unesco, 2015.

VELAZ DE MEDRANO, María Consuelo; MANZANO, Nuria; BLANCO, Ángeles (Dir.). Los procesos de cambio de las políticas públicas sobre orientación y apoyo a la escuela: análisis comparado de sistemas vigentes y emergentes: estudio múltiple de casos en una muestra de Comunidades Autónomas. Madrid: Ministerio de Educación, 2011.

VELAZ DE MEDRANO, María Consuelo et al. El desarrollo profesional de los orientadores de educación secundaria: análisis de necesidades y prospectiva. Revista de Investigación Educativa, Barcelona, v. 19, n. 1, p. 199-220, 2001.

WANG, Huan et al. Can school counseling reduce school dropout in developing countries? Working Paper Rural Education Action Program, Stanford, n. 275, 2014. Disponible en: <https://reap.fsi.stanford.edu/ sites/default/files/275-can_school_counseling_reduce_school_dropout_in_developing_countries.pdf>. Acceso en: 15 my. 2017.

WEISS, Robert. Learning from strangers: the art and method of qualitative interview studies. New York: The Free Prees, 1994.

Recibido en:19.11.2017 Revisiones en: 06.02.2018 Aprobado en: 04.04.2018

David Doncel Abad es profesor ayudante doctor en la Facultad de Educación de la Universidad de Salamanca. Es experto en Sociología de la Educación. Ha participado en varios proyectos de investigación nacionales e internacionales sobre políticas educativas.

Sonsoles San Román es catedrática E.U. de Sociología en la Universidad Autónoma de Madrid. Pionera en el estudio de los procesos de feminización docente con reconocimiento nacional e internacional. Líneas de investigación: representaciones sociales, profesorado, feminización, maestras y contrastes histórico-generacionales.

Carlos Vecina Merchante es doctor en Ciencias de la Educación. Profesor tutor de la UNED y coordinador del Proyecto de Intervención Comunitaria Intercultural (ICI) en el GREC. Sus líneas de investigación se centran en la Intervención social y en la Educación. 